\title{
Trombosis venosa superficial y profunda durante la gestación. Experiencia institucional
}

\author{
María Teresa Peralta Abello; Juan Carlos Sabogal*
}

RESUMEN: Como objetivo general deseamos presentar la experiencia institucional en el manejo médico de la Trombosis Venosa Superficial y Profunda Ante-parto, en el Servicio de Alto Riesgo. Los objetivos específicos se encaminan a conocer la frecuencia, factores relacionados, tipo de lesión y su localización, parámetros para diagnóstico, tratamientos, resultados obstétricos y morbi-mortalidad tanto materna como perinatal. Se trata de un estudio descriptivo con un corte transversal y una sola medición en una muestra de la población, la cual la integra un universo de 6.500 pacientes atendidas desde el 10 . de enero de 1986 al 31 de octubre de 1993. Contamos con 39 gestantes, las cuales nos representan una frecuencia de 6 por 1.000. Encontramos la Superficial asociadas a várices y con tratamiento a base de ASA y reposo la evolución es satisfactoria. En la profunda, la lesión es a nivel Ileo-femoral e izquierda; con anticoagulación plena, previo estudio Doppler, también evolución satisfactoria. Parto a término y por vía vaginal; sin morbi-mortalidad materna ni perinatal.

PALABRAS CLAVES: Trombosis venosa, trombosis superficial, trombosis profunda, Ante-parto, morbi-mortalidad materna y perinatal.

SUMMARY: The general objective of this study was to present the Institutional experience in the medical therapy of the ante-partum superficial and deep venous thrombosis in the High-Risk Pregnancy Service of the Instituto Materno Infantil of Bogotá. The specific objectives were to identify the frequency, associated factor, type and localization of the lesion, diagnostic criteria, treatment, obstetric outcome and maternal and perinatal morbidity and mortality. This is a descriptive cross-sectional study which universe consisted of 6.500 patients who assisted to de Service from January 1st, 1986 to october 31, 1993. We found 39 patients with venous thrombosis, resulting in a 61/000 incidence. Superficial venous thrombosis was associated with varices and the treatment with ASA and bed rest was effective. Deep venous thrombosis affected mainly the ileo-femoral territory. After a Doppler study, the therapeutic anticoagulation was also effective. Vaginal delivery occurred at term withouth maternal or perinatal morbidity and mortality.

KEY WORDS: Venous thrombosis, Superficial thrombosis, deep thrombosis, pregnancy, maternal and perinatal morbidity and mortality.

\section{Introducción}

La Enfermedad Tromboembólica, es una enfermedad cuya mortalidad ha sido menospreciada en nuestro medio.

El embarazo durante largo tiempo ha sido reconocido como un estado en el cual ocurren fenómenos que causan hipercuagulabilidad, tales como la estasis venosa, niveles aumentados de factores de coagulación y disminución de la actividad fibrinolítica. Ocurre además, una disminución del tono venoso dependiente del influjo hormonal predominante y del retorno venoso a partir del sistema venoso de los miembros inferiores; lo anterior es consecuencia de la presión causada por el útero grávido sobre la cava y la vena ilíaca izquierda.

El fibrinógeno y los factores VII, X, VIII; C y VIII $\mathrm{Ag}$, aumentan, por lo tanto el tiempo de protrombina y el parcial de tromboplastina se acortan; la generación de trombina también se aumenta.

\footnotetext{
* Servicio Alto Riesgo. Instituto Materno Infantil. Departamento de Obstetricia y Ginecología. Facultad de Medicina. Universidad Nacional de Colombia.
}

La incidencia de las entidades varía entre 1.9 por 1.000 a 2.9 por 1.000 durante la gestación; y entre 1 y 10 por 1.000 en el puerperio. La complicación de la Trombosis Profunda relacionada con el Tromboembolismo Pulmonar podría ser en un $24 \%$.

El fenómeno inicial en el génesis de la Trombosis Profunda parece ser el daño endotelial, causado por la turbulencia de las áreas venosas adyacentes a las válvulas del sistema venoso de los miembros inferiores. La lesión valvular propicia la agregación y depósito de acúmulos de plaquetas las cuales liberan sustancias trombogénicas, que llevan a la formación del trombo.

Es posible enumerar varios factores que han sido relacionados con la Trombosis Profunda; algunos fármacos utilizados para inhibir la lactancia, la edad, la multiparidad, trauma o várices.

Clínicamente se asocian la presencia de dolor, edema, diferencia de diámetros de más de 2 centímetros con el miembro contralateral; dolor muscular y signo de Homans. El método ideal para confirmar diagnóstico es el Doppler con ultrasonido de tiempo real; no invasivo, práctico y confiable.

El tratamiento se encamina a evitar el desarrollo del Tromboembolismo Pulmonar. La anticoagulación plena 
con heparina durante 7 a 14 días, ( 24 a 30.000 unidades en 24 horas) y profilaxis posterior con 10.000 unidades sub-cutáneas, es lo recomendado.

Deseamos comunicar la situación de las pacientes con diagnóstico de Trombosis Venosa Profunda y Superficial Ante-Parto, desde el momento del ingreso al Servicio de Alto Riesgo, condiciones materno-fetales; factores relacionados, tratamientos y morbi-mortalidad materna y perinatal. Con los resultados obtenidos se espera presentar el beneficio de la conducta de manejo, basada en los siguientes parámetros:

- Normas generales para paciente hospitalizada en Alto Riesgo; vigilancia de la unidad feto-placentaria y reposo en cama.

- Ante Trombosis Superficial además del reposo se recomienda ASA 300 mgrs. en 24 horas. Medias de soporte o vendaje al salir.

- Confirmar diagnóstico de Trombosis Profunda con estudio Doppler e instaurar anticoagulación plena con Heparina, 24.000 unidades durante 10 días, con paciente hospitalizada y controles estrictos de tiempo de protrombina y parcial de tromboplastina. Profilaxis subcutánea con 10.000 unidades hasta la semana 37. Reanudar la profilaxis en el puerperio durante 3 semanas.

- Continuar en control prenatal y esperar parto a término y por vía vaginal. La operación Cesárea por indicaciones obstétricas.

- No anticoncepción hormonal y profilaxis con heparina en futuras gestaciones.

Propósitos. Evaluar la Trombosis Superficial y Profunda en el Servicio de Alto Riesgo bajo diferentes aspectos.

Objetivo general.Presentar la experiencia institucional en el tratamiento médico de la Trombosis Superficial y Profunda Ante-parto.

\section{Objetivos específicos}

- Llevar a cabo la revisión de historias clínicas de todas las pacientes con diagnóstico de Trombosis Superficial y Profunda atendidas en el Servicio de Alto Riesgo del Instituto Materno Infantil.

- Conocer la frecuencia, algunos factores relacionados como edad, paridad, edad gestacional y condiciones al ingreso.

- Establecer el tipo de lesión, en cuanto a localización, Superficial o Profunda, derecha o izquierda.

- Determinar parámetros clínicos para el diagnóstico y la confirmación con métodos paraclínicos.

- Conocer tratamientos instaurados, conductas obstétricas, momento y vía del parto; indicaciones de la operación cesárea y condiciones de los recién nacidos.

- Determinar las causas de morbi-mortalidad materna y perinatal; frecuencia del Tromboembolismo Pulmonar y establecer los promedios de estadía.

- Identificar para cada paciente las variables antes mencionadas.

\section{Materiales y métodos}

Tipo de estudio. La presente investigación corresponde a un estudio descriptivo con un corte transversal y una sola medición en una muestra de la población.

\section{Población de estudio}

1. Ubicación Geográfica: Pacientes atendidas en el Servicio de Alto Riesgo del Instituto Materno Infantil.

2. Universo: 6.500 pacientes atendidas desde el 10 . de enero de 1986 al 31 de octubre de 1993.

3. Muestras: 39 Gestantes manejadas en el Servicio de Alto Riesgo en el mismo período.

\section{Recolección de la información}

Técnica de recolección mediante la revisión de historias clínicas y archivo privado de Alto Riesgo.

\section{Procesamiento de la información}

Los datos se tabularán en un Computador PC 386.

\section{Análisis de la información}

Se utilizará análisis descriptivo a través de distribución de frecuencias, porcentajes, promedios e índices.

\section{Presentación de resultados}

En forma narrativa, tablas, cuadros y gráficas.

\section{Resultados}

\section{Movimiento del Servicio de Alto Riesgo}

Durante el período comprendido entre el 1o. de enero de 1986, fecha en la cual se inició la reorganización del Servicio de Alto Riesgo anteparto, hasta el 31 de octubre de 1993 se han atendido aproximadamente 6.500 embarazadas, las cuales corresponden al $7.4 \%$ de los ingresos del Instituto Materno Infantil. Con un promedio de atención de 100 pacientes nuevas por mes, 600 partos al año, ( $26 \%$ pretérminos), una frecuencia de cesáreas del $37.6 \%$ y de abortos del $17.6 \%$.

El 84\% de los ingresos se relacionan con complicaciones propias de la gestación: amenaza de parto pretérmino, embarazo prolongado, cesárea anterior, hipertensión gravídica, hemorragias, embarazo múltiple, RPM. El 15\% representa las complicaciones médicas: infecciones de vías urinarias, síndromes convulsivos, diabetes, cardiopatías. El $1 \%$ restante corresponde a complicaciones quirúrgicas: tumores torcidos de ovario, trauma, apendicitis. Con un índice general de morbilidad materna del $5.6 \%$, debido en primera instancia a la Endometritis Postparto (embarazo prolongado, hipertensión, hemorragias) y en cuanto a mortalidad materna un índice de 3.4 por 1.000 representado por causas no obstétricas: cáncer, tromboembolismo pulmonar. La mortalidad perinatal depurada a un 52 por 1.000 con el pretérmino ocupando 
los primeros lugares tanto en la anteparto como en la neonatal precoz. Los promedios de estadía son de 5.8 días, con un porcentaje de ocupación del $89.5 \%$ para 24 camas disponibles.

\section{Frecuencia}

En el grupo de las complicaciones médicas durante la gestación, la trombosis venosa de miembros inferiores, tiene una frecuencia de 6 por 1.000 ; ocupando el séptimo lugar como motivo de ingreso al servicio.

Presentamos 39 gestantes, de las cuales, el $66.7 \%$ (26), tienen diagnóstico de trombosis profunda y el $33.3 \%$ (13), de trombosis venosa superficial. Tendríamos entonces, para la primera una frecuencia sobre el total de embarazadas atendidas de 4 por 1.000 y para la segunda de 2 por 1.000 . Cuatro y dos pacientes nuevas por año respectivamente.

\section{Factores relacionados}

En cuanto a edad, paridad y momento del diagnóstico encontramos los siguientes promedios: 26.9 años (mínimo 16 y máximo 40); 2.5 hijos (mínimo 1 y máximo 7) y consultan hacia las 27.5 semanas, con un mínimo hacia las 8 y máximo hacia las 40 .

No hay diferencia en estos factores relacionados si se trata de una superficial o de una profunda. Por ejemplo, en la paridad: para la profunda en promedio 2 hijos (mínimo 1, máximo 6) y en la superficial 3 hijos (mínimo 1, máximo 7).

\section{Condiciones al ingreso y manejo}

La sintomatología dolorosa hacia la fosa ilíaca e irradiada hacia el miembro inferior comprometido, con dificultad para la marcha, claudicación e iniciación súbita, caracterizaron el motivo de consulta en todas las gestantes con trombosis profunda. Los hallazgos clínicos demostraron edema, signo de Homans y aumento de los diámetros en relación con el miembro inferior contralateral.

Aquellas con antecedente de várices, refieren sus síntomas dolorosos también hacia el lado comprometido. Se encuentra rubor, edema y dolor con evidencia de cambios en las zonas afectadas.

De acuerdo con las normas del servicio, se hospitalizaron, se establecieron condiciones materno-fetales y la presencia de otras patologías sobreagregadas.

Instaurado el reposo en cama, ante trombosis superficial se indicó la terapia con ASA (300 mgrs. por día) al dar la salida, una vez controlada la fase aguda, se recomendaron medias de soporte o los vendajes elásticos.

Con sospecha clínica de trombosis profunda, se solicitó el estudio con Doppler en todas las pacientes e inmediatamente se ordenó la terapia con Heparina por vía endovenosa ( 24.000 unidades en 24 horas). Según las disponibilidades de la unidad, con infusión continua o cada 4 horas y durante 10 días con gestante hospitalizada. Controles de TP y TPP. Al darlas de alta para continuar en la consulta de prenatal, se instruyeron para la autoaplicación de la profilaxis del medicamento hasta la semana 37. Al reingresar, en el post-parto, se le reanudo por 3 semanas más. Gráfica 1.

\section{Gráfica 1 \\ TROMBOSIS VENOSA EN LA GESTACION. LOCALIZACION DE LA LESION}

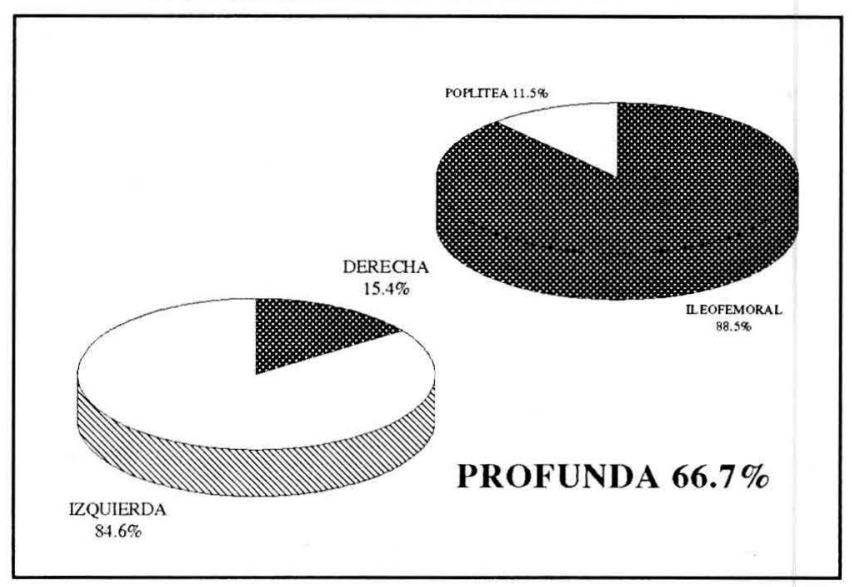

\section{Localización de la lesión}

El $84.6 \%$ (22 casos) de las lesiones en trombosis profunda, se localizaron en el lado izquierdo, y el porcentaje restante $15.4 \%$ en el lado derecho de la gestante. En cuanto al sitio de la misma, tanto la sintomatología clínica como los resultados paraclínicos, confirmaron: 1. Ileofemoral en 23 pacientes, $88.5 \%$, (22 izquierdas, $95.6 \%$ y 1 derecha $4.4 \%$ ). 2. Poplítea en 3 pacientes, $11.5 \%$; otras del lado derecho.

Predomina, para resumir entonces, la trombosis Ileofemoral izquierda. Gráfica 1.

En las 13 gestantes con diagnóstico de trombosis superficial encontramos compromiso por várices en forma bilateral en $6(46.1 \%)$, izquierdas $4(30.7 \%)$ y derechas $3(23.0 \%)$. Igualmente encontramos mayor compromiso hacia el lado izquierdo. Gráfica 2.

\section{Gráfica 2}

TROMBOSIS VENOSA EN LA GESTACION. LOCALIZACION DE LA LESION

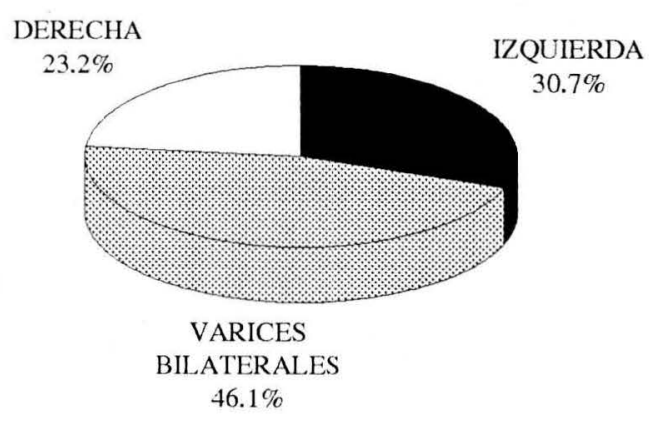

SUPERFICIAL $\mathbf{3 3}, \mathbf{3 \%}$

\section{Momento y vía del parto}

Todas las gestaciones llegaron al término; por lo tanto no reportamos abortos, ni partos pre-términos en esta serie. 
Al momento del análisis de estos resultados tenemos dos embarazadas en control prenatal, con antecedente de trombosis venosa profunda íleo-femoral izquierda.

El promedio del inicio espontáneo del trabajo de parto, es hacia las 38.8 semanas de gestación, con un mínimo a las 37 y un máximo a las 41 . Igual para la superficial que para la profunda.

E1 84.6\% (33 pacientes) se atendieron por vía vaginal sin complicaciones ( 21 profundas y 12 superficiales).

Las indicaciones de la operación cesárea en las 4 restantes $(15.4 \%)$, fueron por Presentación de Pelvis (3 pacientes, $75 \%$ ) y antecedentes de cesáreas en el $25 \%$ con una paciente.

La entidad de base determinó desembarazar por complicaciones maternas o fetales. Gráfica 3.

\section{Gráfica 3}

TROMBOSIS VENOSA EN LA GESTACION. MOMENTO Y VIA DEL PARTO. 38.8 SEM.

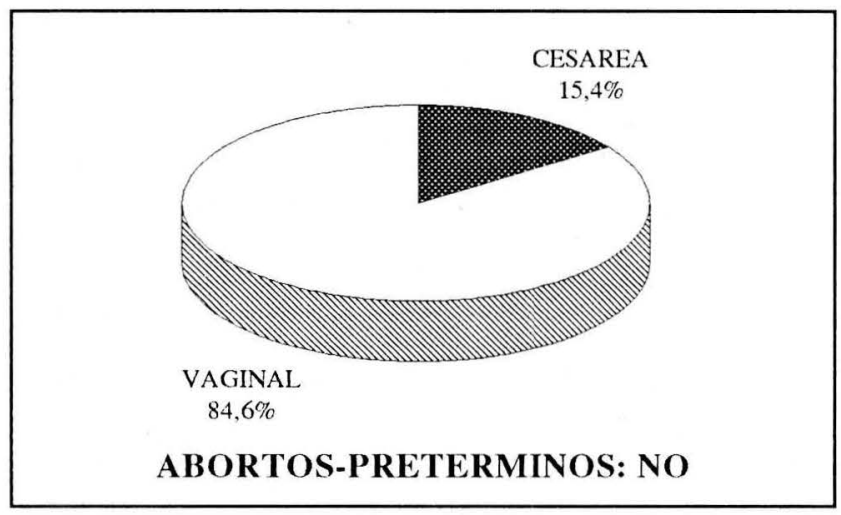

\section{Condiciones de los recién nacidos}

Los 19 recién nacidos de sexo masculino (51.4\%) y los 18 de sexo femenino (48.6\%), fueron calificados con Apgar a los 5 minutos de $10 / 10$, y en cuanto a peso se refiere, el mínimo fue de 2.600 gramos y el máximo de 4.000 gramos, para un promedio de 3.247 gramos.

\section{Morbi-mortalidad materna}

No reportamos ningún caso de tromboembolismo pulmonar relacionado con la trombosis profunda; tampoco complicaciones con la anticoagulación u otro tipo de morbilidad propia de la gestación.

Para esta serie no presentamos mortalidad materna.

\section{Morbi-mortalidad perinatal}

La trombosis venosa profunda o superficial no inciden en la morbi-mortalidad perinatal durante el período agudo de la enfermedad. Los índices para este grupo son de cero y todos los recién nacidos evolucionaron satisfactoriamente.

\section{Estadía}

En los promedios de estadía, la trombosis profunda sobrepasa a la superficial con 13.1 y 8.5 días de hospitalización (mínimos de 10 y 5 , con máximos de 30 y 14 días). (Cuadro 1).

$$
\text { Cuadro } 1
$$

TROMBOSIS VENOSA DURANTE LA GESTACION

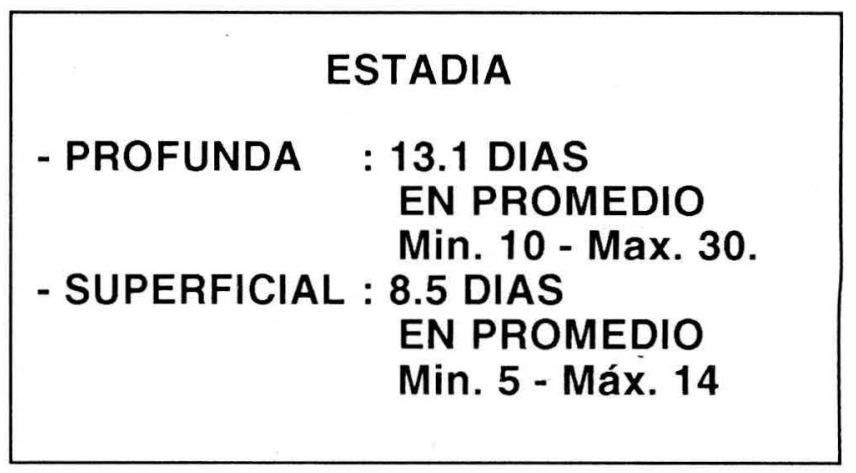

En general el promedio es de 11.5 días. (Mínimo 5 y máximo 30).

\section{Discusión de resultados}

La experiencia institucional en el Servicio de Alto Riesgo del Instituto Materno Infantil, en el manejo de la trombosis venosa profunda y superficial, nos ha permitido conocer el beneficio de un diagnóstico y un tratamiento oportuno y su relación con la mejoría clínica y los resultados satisfactorios en cuanto a morbi-mortalidad materna y perinatal se refiere.

Es importante la frecuencia de la entidad anteparto, en el puerperio no la tenemos; el compromiso íleo femoral y sin tromboembolismo pulmonar.

\section{Recomendaciones}

Continuar los parámetros establecidos en el Servicio para su diagnóstico y tratamiento.

\section{BIBLIOGRAFIA}

Moser KM. Venus Tromboembolism. Am. Rev. Resp. Disease. 1990; 141: 235-249.

Hull R., Hish J et al. Pulmonary Angiography, ventilation lug scanning and venography por clinically suspected pulmonary embolism with abnormal perfusion scans. Anns. Intern. Med. 1983; 98:891-899.

Creasy R., Resnik R. Maternal Fetal Medicine: principle and practice, 2end edition. WB Saunders Company, Philadelphia, 1989.

Demer C., Ginsberg JS. Deep Venous Thrombosis and Pulmonary Embolism in Pregnancy. Clin. Chest. Med. 1992; 13: 645-655.

Seviit S. Pathology and Pathogenesis of Deep Vein Thrombi. En: Moser KM, Stein M, Pulmonary Thromboembolsim Chicago, Year Book Publishers, 1973; 93-103.
Wessler S. Medical Management of Venous Thrombosis. Ann. Rev. Med. 1976; $27: 313$.

Hull R., Taylor DW., Hirsh J et al. Impedance Plethysmography: The relationship between venous filling and sensitivity and specificity for proximal vein thrombosis. Circulation, 1978; 58: 896-906.

Hirsh J. Blood testes for the diagnosis of venous and arterial thrombosis. Blood, 1981; 57: 1, 1 .

Salzman E., Deykin D., Shapiro R et al. Management of heparin therapy: Controlled prospective trial. New. Engl. J. Med. 1975; 292: 1048. 